\section{Military Technical College Kobry El-Kobbah, Cairo, Egypt}

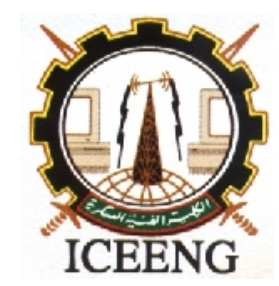

\section{$8^{\text {th }}$ International Conference on Electrical Engineering \\ ICEENG 2012}

\title{
Empirical Mode Decomposition Complexity
}

$$
\text { By }
$$

\author{
Sherif Elgamel *
}

\section{$\underline{\text { Abstract: }}$}

Empirical mode decomposition (EMD) was developed for analyzing non-linear and non-stationary data. EMD decomposition is based on the local characteristic time scale of data. EMD decomposes any data set into a finite and often small number of intrinsic mode functions (IMF). An IMF is defined as any function having the same numbers of zero crossings and extrema, and also having symmetric envelopes defined by the local maximal and minima, respectively. The IMF also admits well behaved Hilbert transform verified to be highly orthogonal. EMD is used in many applications such as signal enhancements and data analysis. In this paper, the EMD is presented using computer simulations. The complexity of classical EMD is calculated to determine the additive complexity to any system uses the EMD.

\section{Keywords:}

Empirical mode decomposition, Intrinsic Mode Functions, and systems complexity.

* Electronic Warfare Department, Military Technical College, Cairo, Egypt 


\section{Introduction:}

Empirical mode decomposition (EMD) is Empirical because it lacks theoretical foundations. Mode relates to the intrinsic mode functions which represent the oscillation modes embedded in the data. Decomposition refers to decomposing the original signal to IMFs and residual.

The idea of EMD, which is also known as the Hilbert Huang transformation (HHT), appeared in 1998 [1] and was the winner of the 2002 NASA Government Invention of the Year [2]. It is recognized by NASA Headquarters inventions and contributions board as "One of the most important discoveries in the field of applied mathematics in NASA history". Norden E. Huang who works for NASA Goddard Space Flight Center reviewed the non-stationary data processing methods as the spectrogram, wavelet analysis, Wigner-Ville distribution, evolutionary spectrum, empirical orthogonal function expansion (EOF), and other miscellaneous methods, and then showed the superior performance of EMD over these methods [3].

EMD is used in many applications such as: nonlinear wave evolution, climate cycles, earthquake engineering, submarine design, structural damage detection, satellite data analysis, turbulence flow, blood pressure variations and heart arrhythmia, nondestructive testing, structural health monitoring, signal enhancement, or economic data analysis [4-7].

In this paper, due to the wide use of EMD in many applications, the EMD complexity is calculated to determine the overall systems complexity due to the usage of EMD.

The remainder of the paper is organized as follows. In Section 2, the classical EMD and its decomposition algorithm is introduced. In Section 3, an example to understand exactly how the classical EMD algorithm works is provided. In Section 4, the complexity for classical EMD is presented. The paper is concluded in section 5.

\section{Empirical mode decomposition}

\section{Classical EMD}

EMD is a non-linear technique for analyzing and representing non-stationary signals [8]. EMD is data-driven and decomposes a time domain signal $x[n]$ into a complete and finite set of adaptive basis functions which are defined as IMFs, $\boldsymbol{h}^{(i)}[n], 1 \leq i \leq L_{I M F}$. These IMFs are not predefined as in the case with the Fourier and the Wavelet transforms. The IMFs are oscillatory and have no DC component [9-11], so the signal $\boldsymbol{x}[n]$ can be represented as

$$
\boldsymbol{x}[n]=\sum_{i=1}^{L_{M F}} \boldsymbol{h}^{(i)}[n]+\boldsymbol{d}[n]
$$

where $\boldsymbol{d}[n]$ is the residual. 
When a signal $\boldsymbol{x}[n]$, that comprises a slowly oscillation relative to the sampling frequency superimposed on a highly oscillation signal relative to the sampling frequency (in our case additive interference noise signal), is applied to the EMD algorithm, the first IMFs tend to contain the highly oscillation signal (noise) and the remaining IMFs contain the useful signal. In applications such as low frequency noise interference, the first IMFs in the EMD decomposition are the useful signal and the remainder contains the main noise components. Thus apriori knowledge of the noise characteristics in the EMD decomposed signal structure can be used to obtain the best performance.

Each IMF is estimated with the aid of an iterative process called sifting that is applied to the residual multi-component signal as shown in Fig. 1.

$$
\boldsymbol{x}^{(i)}[n]= \begin{cases}\boldsymbol{x}[n] & i=1 \\ \boldsymbol{x}[n]-\sum_{q=1}^{i-1} \boldsymbol{h}^{(q)}[n] & i \geq 2\end{cases}
$$

During the $(m+1)^{\text {th }}$ sifting iteration, the temporary IMF estimate $\boldsymbol{h}_{m}{ }^{(i)}[n]$ is improved according to the following steps:

Table 1: Temporary IMF estimate $\boldsymbol{h}_{m}{ }^{(i)}[n]$ improving

1 While $\boldsymbol{h}_{m}{ }^{(i)}[n]-\boldsymbol{M}_{m}[n]$ is not sufficiently close to zero

2 Identify all extrema (maxima and minima) of $\boldsymbol{h}_{m}{ }^{(i)}[n]$.

3 Interpolate the extrema points of $\boldsymbol{h}_{m}{ }^{(i)}[n]$ to make upper and lower envelope.

4 Compute the mean $\boldsymbol{M}_{m}[n]$ of the two envelopes (upper and lower).

5 Obtain the refined estimate $\boldsymbol{h}_{m+1}{ }^{(i)}[n]$ of the IMF by subtracting the mean found in the previous step from the current IMF estimate $\boldsymbol{h}_{m}{ }^{(i)}[n]-\boldsymbol{M}_{m}[n]$.

\section{End}




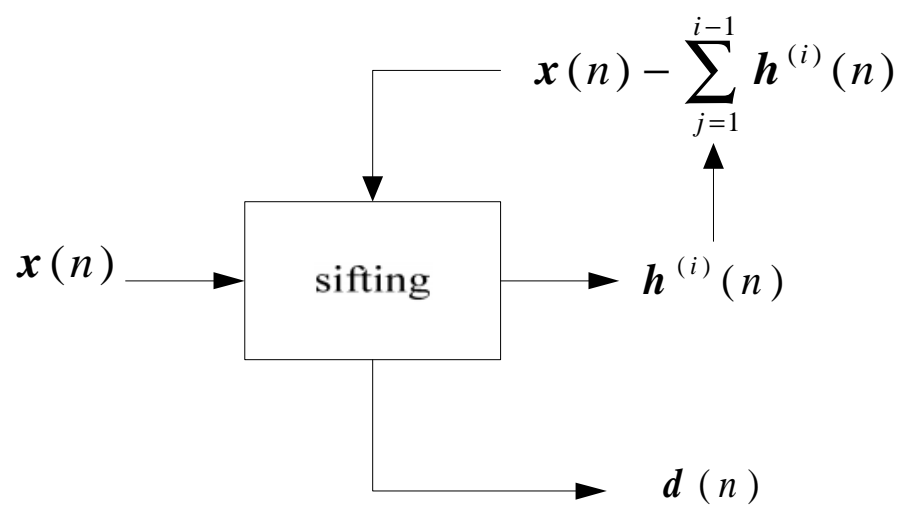

Fig. 1 The EMD sifting process

\section{Complexity Calculation of the Classical EMD}

To understand exactly how the classical EMD algorithm works, the signal $\boldsymbol{x}[n]$ consists of the linear chirp signal with the following parameters :- starting frequency of $5 \mathrm{~Hz}$, stopping frequency of $100 \mathrm{~Hz}$, chirp period of $0.8 \mathrm{sec}$, and sampling frequency of 1 $\mathrm{kHz}$; time window $5 \mathrm{sec}$, and the chirp start at $1.5 \mathrm{sec}$ shown in Fig.2-A added to a sinusoidal signal $\sin (200 \pi t$ ) with sampling frequency $1000 \mathrm{~Hz}$ (shown in Fig.2-B) to form the combined signal shown in Fig.2-C .

Fig.3-A shows the interpolation for all maxima points and all the minima points of the temporarily IMF $\boldsymbol{h}_{m}{ }^{(i)}[n]$ on two different curves. Fig.3-B shows a zoomed version of Fig. 7-A.
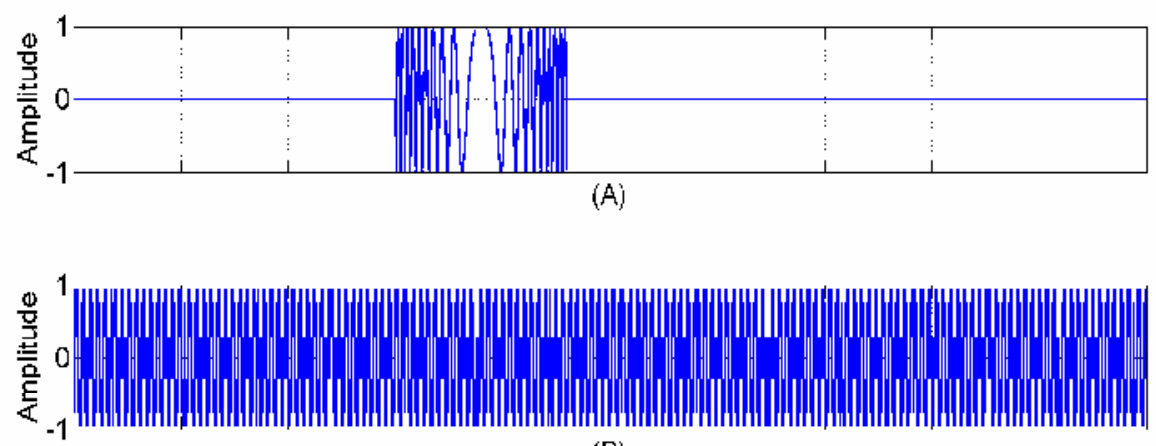

(B)

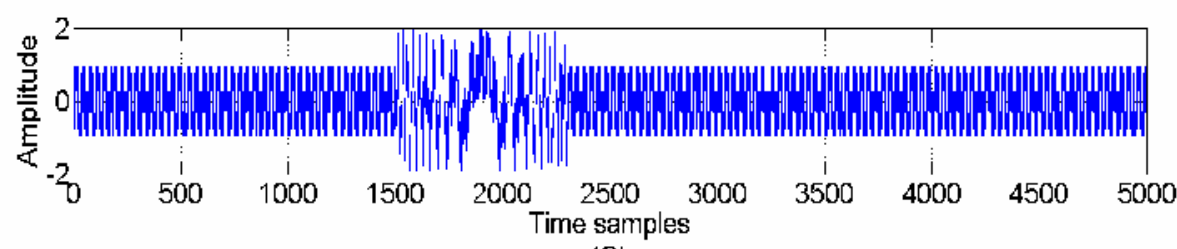

(C)

Fig.2 Combined chirp with sinusoidal signals 
The mean curve $\boldsymbol{m}_{m}[n]$ is calculated as the mean of the interpolated curves and it is shown in Fig.3-C. The extracted mean $\boldsymbol{m}_{m}[n]$ is subtracted from the original $\boldsymbol{x}[n]$ and whole sifting iteration process continues for $m$ times until the mean $\boldsymbol{m}_{m}[n]$ is reaching the stopping threshold criteria with nearly zero-mean. $\boldsymbol{m}_{m}[n]$ reaches the stoping criteria at the $m+1$ iteration process and the temporarily IMF $\boldsymbol{h}_{m}{ }^{(i)}[n]$ is considered now as the $1^{\text {st }} \operatorname{IMF} \boldsymbol{h}^{1}[n] . \boldsymbol{h}^{1}[n]$ is shown in Fig. 4 and contains nearly all the sinusoidal signal $\sin (200 \pi t)$ due to the fact that the first IMFs always contain the signal's high frequency components. The sifting process starts again with input signal $\boldsymbol{x}[n]-\boldsymbol{h}^{1}[n]$. All the previous processing work is repeated until the number of maxima points or minima points equal one (interpolation must be done at least between two points) and the last IMF (IMF 9) is achieved as seen in Fig. 4. Note that the sum of the extracted IMFs from the $1^{\text {st }}$ IMF $1 \boldsymbol{h}^{1}[n]$ to the last IMF $9 \boldsymbol{h}^{9}[n]$ results in the combined signal $\boldsymbol{x}[n]$.

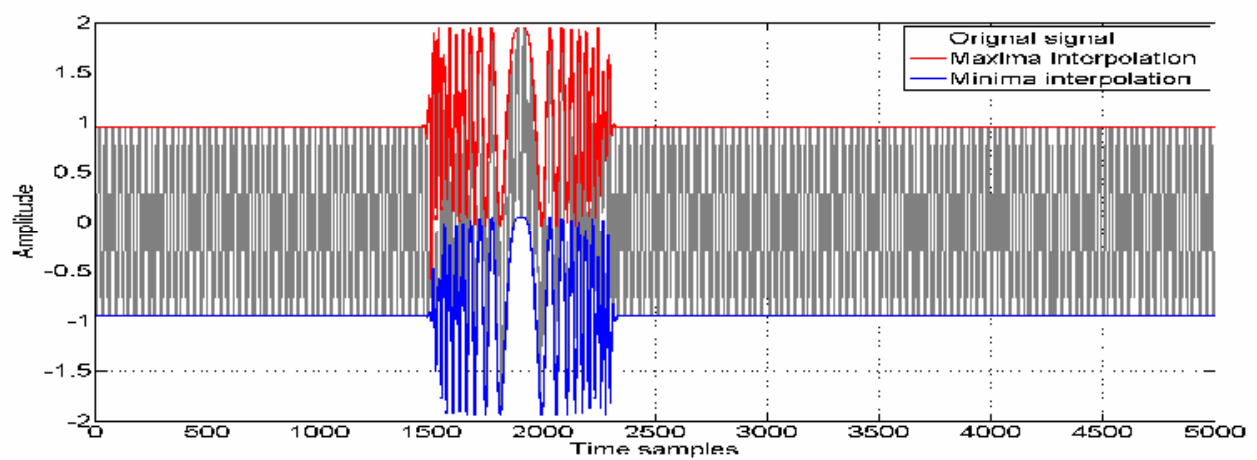

(A)

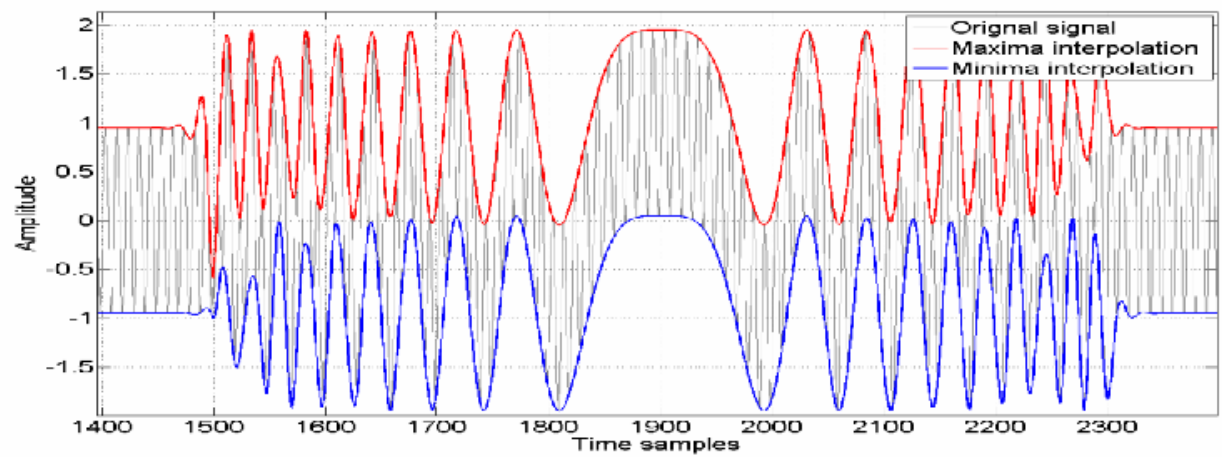

(B) 


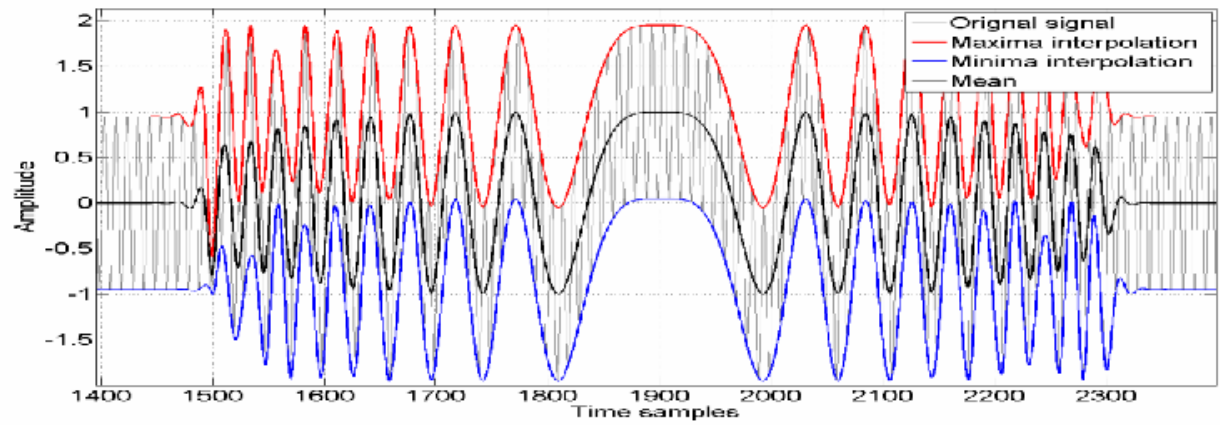

(C)

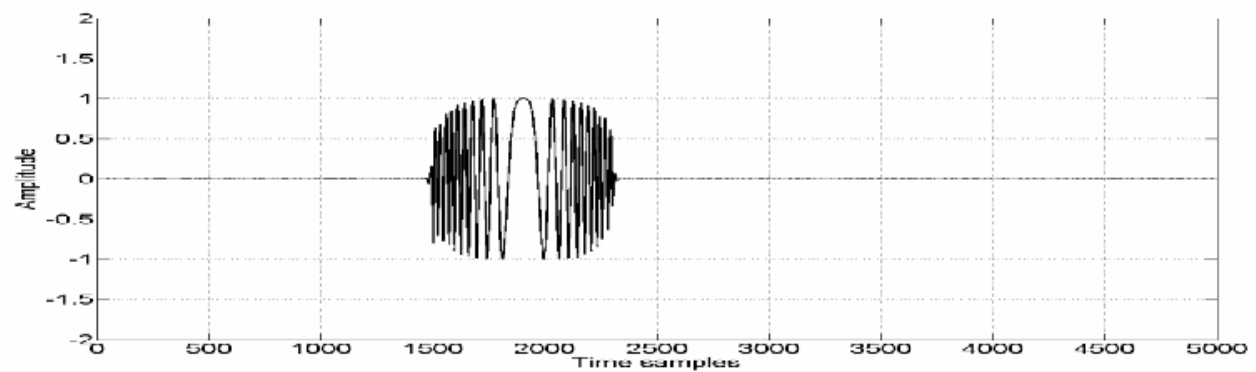

(D)

Fig.3 Classical EMD algorithm steps
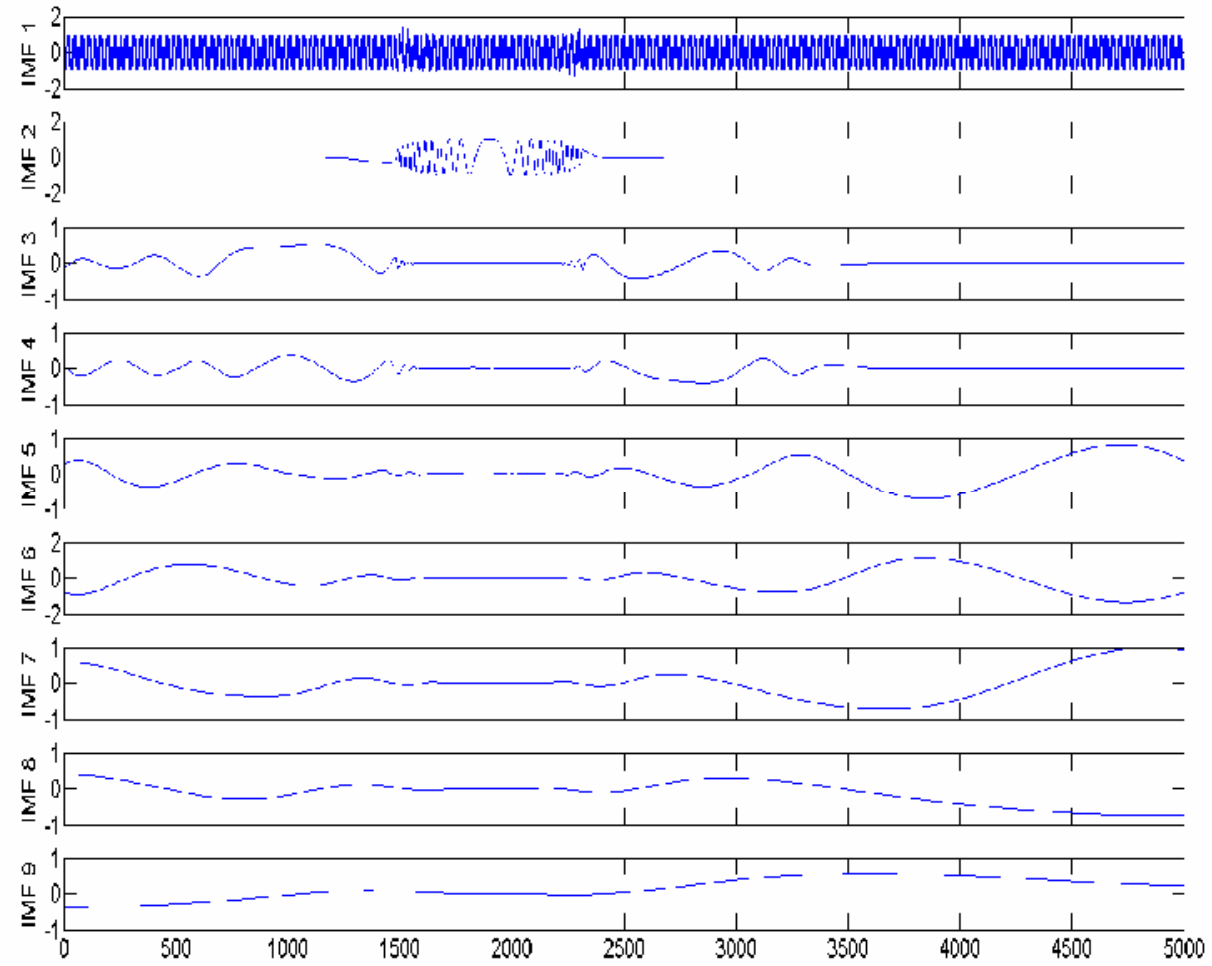

Fig. 4 The IMFs of the combined signal 


\section{Complexity Calculation of the Classical EMD}

The complexity of the classical EMD is calculated using the EMD algorithm shown in Table 1. The total EMD complexity calculation is shown in Table 2.

In Table 2, to identify all extrema (maxima and minima) of $\boldsymbol{h}_{m}{ }^{(i)}(n)$, it is needed nearly $N$ operations for the subtraction and $\frac{N}{2}$ at maximum for the comparisons with $\boldsymbol{M}_{m}[n]$, where $N$ is the number of samples of the decomposed signal. Six multiplication and three addition operations are needed to interpolate the extrema points of $\boldsymbol{h}_{m}{ }^{(i)}(n)$ to make upper or lower envelope using cubic spline interpolation [12]. Thus the complexity of this cubic spline interpolation can be approximated to $6 \mathrm{~N}$ and it is duplicated to $12 N$ to make the upper and lower envelope. $N$ computational cost is needed to compute the mean $\boldsymbol{M}_{m}(n)$ of the two envelopes (upper and lower) due to the additive operation. The refined estimate $\boldsymbol{h}_{m+1}{ }^{(i)}(n)$ needs $N$ operation due to the subtraction as shown in step 4 in Table 2. The difference $\boldsymbol{h}_{m}{ }^{(i)}(n)-\boldsymbol{M}_{m}(n)$ is repeated $Q$ times until this difference can be considered as zero mean and fulfilled a stopping criterion. $Q$ take the value of 8 , at the $8^{\text {th }}$ loop this difference reaches nearly the value of zero mean[13, 14]. Using classical EMD algorithms the maximum number of these IMFs is $O\left(\log _{2} L\right)[15,16]$. Thus the total complexity for the classical EMD can be $\begin{array}{lllllll}\text { calculated as } & \text { seen }\end{array}$ as $\log _{2} N\left(Q\left(N+12 N+N+N+\frac{N}{2}\right)\right) \approx \log _{2} N\left(Q\left(15 N+\frac{N}{2}\right)\right.$.

\section{Conclusion}

In this paper, the EMD is presented using computer simulations. The complexity of classical EMD is calculated to determine the additive complexity to any system uses the EMD. It is found that the classical EMD complexity can be approximated to $\log _{2} N\left(Q\left(15 N+\frac{N}{2}\right)\right.$, where $N$ is the number of samples of the decomposed signal and $Q$ is a constant take the value of 8 . 
Table 2: EMD complexity calculation

\begin{tabular}{|c|c|c|}
\hline no & EMD steps & Complexity \\
\hline 1 & $\begin{array}{l}\text { Identify all extrema (maxima and minima) } \\
\text { of } \boldsymbol{h}_{m}{ }^{(i)}(n)\end{array}$ & $\begin{array}{l}\approx N(\text { subtraction }) \\
+\frac{N}{2} \text { at max comparisons }\end{array}$ \\
\hline 2 & $\begin{array}{l}\text { Interpolate the extrema points of } \\
\boldsymbol{h}_{m}{ }^{(i)}(n) \text { to make upper and lower } \\
\text { envelope. }\end{array}$ & $\begin{array}{l}2 * 6 N \quad(6 \text { multiplication } \\
\text { addition) for cubic } 3 \\
\text { interpolation [12] }\end{array}$ \\
\hline 3 & $\begin{array}{l}\text { Compute the mean } \boldsymbol{M}_{m}(n) \text { of the two } \\
\text { envelopes (upper and lower). }\end{array}$ & $N$ (addition) \\
\hline 4 & $\begin{array}{l}\text { Obtain the refined estimate } \boldsymbol{h}_{m+1}{ }^{(i)}(n) \text { of } \\
\text { the IMF by subtracting the mean found in } \\
\text { the previous step from the current IMF } \\
\text { estimate } \boldsymbol{h}_{m}{ }^{(i)}(n)-\boldsymbol{M}_{m}(n) \text {. }\end{array}$ & $N$ (subtraction) \\
\hline 5 & $\begin{array}{l}\text { Repeat the loop from } 1 \text { to } 4 \text { until } \\
\boldsymbol{h}_{m}{ }^{(i)}(n)-\boldsymbol{M}_{m}(n) \text { can be considered as } \\
\text { zero mean and fulfilled a stopping } \\
\text { criterion. }\end{array}$ & $\begin{array}{l}Q \text { times (can be considered as } 8 \text { ) } \\
\text { Considering the IMF iteration reach } \\
\text { zero mean at the } Q^{\text {th }} \text { iterations }[13 \text {, } \\
14]\end{array}$ \\
\hline 6 & Max no of IMFs & $\log _{2} N$ \\
\hline 7 & Total complexity & $\begin{array}{l}\log _{2} N\left(Q\left(N+12 N+N+N+\frac{N}{2}\right)\right. \\
\approx \log _{2} N\left(Q\left(15 N+\frac{N}{2}\right)\right.\end{array}$ \\
\hline
\end{tabular}

\section{$\underline{\text { References }}$}

[1] N. E. Huang, et al., "The empirical mode decomposition and the Hilbert spectrum for nonlinear and non-stationary time series analysis," Proc. R. Soc. London, vol. Ser. A, 454, pp. 903-995, 1998.

[2] NASA Inventions and Contributions Board Available: http://www.nasa.gov/offices/oce/icb/winners/ioy/2002_ioy.html

[3] N. E. Huang, et al., "A confidence limit for the empirical mode decomposition and Hilbert spectral analysis," Procedures of the Royal Society of London, vol. 459, pp. 2317-2345, 2003. 
[4] B. Narsimha, et al., "Denoising and QRS detection of ECG signals using Empirical Mode Decomposition," in Communications and Signal Processing (ICCSP), 2011 International Conference on, 2011, pp. 439-442.

[5] H. Ji, et al., "Flow Pattern Identification Based on EMD and LS-SVM for Gas-TwoPhase Flow in a Minichannel," Instrumentation and Measurement, IEEE Transactions on, vol. 60, pp. 1917-1924, 2011.

[6] N. ur Rehman and D. P. Mandic, "Filter Bank Property of Multivariate Empirical Mode Decomposition," Signal Processing, IEEE Transactions on, vol. 59, pp. 24212426, 2011.

[7] A. Roy and J. F. Doherty, "Overlay Communications Using Empirical Mode Decomposition," Systems Journal, IEEE, vol. 5, pp. 121-128, 2011.

[8] A. D. Lanterman, et al., "Wide-angle radar imaging using time-frequency distributions," Radar, Sonar and Navigation, IEE Proceedings -, vol. 150, pp. 203$11,2003$.

[9] T. Tanaka and D. P. Mandic, "Complex Empirical Mode Decomposition," Signal Processing Letters, IEEE, vol. 14, pp. 101-104, 2007.

[10] G. Rilling, et al., "Bivariate Empirical Mode Decomposition," Signal Processing Letters, IEEE, vol. 14, pp. 936-939, 2007.

[11] Y. Kopsinis and S. McLaughlin, "Development of EMD-Based Denoising Methods Inspired by Wavelet Thresholding," Signal Processing, IEEE Transactions on, vol. 57, pp. 1351-1362, 2009.

[12] A. Mitra and H. S. Dhillon, "Evaluating Sinusoidal Functions by a Low Complexity Cubic Spline Interpolator with Error Optimization," International Journal of Electrical and Computer Engineering, vol. 2, 2007.

[13] G. Rilling and P. Flandrin, "One or Two Frequencies? The Empirical Mode Decomposition Answers," Signal Processing, IEEE Transactions on, vol. 56, pp. 85-95, 2008.

[14] P. Flandrin, et al. Empirical Mode Decomposition [Online]. Available: http://perso.ens-lyon.fr/patrick.flandrin/emd.html

[15] P. Flandrin, et al., "Detrending and denoising with Empirical Mode Decompositions," presented at the The 2004 European Signal Processing Conference (EUSIPCO-2004), 2004

[16] P. Flandrin, et al., "EMD Equivalent Filter Banks, from Interpretation to Applications," in Hilbert-Huang Transform and Its Applications ed: World Scientific, 2005, pp. pp. $57-74$. 